Ann. Zootech., I964, 13 (2), I5I-I54.

\title{
TROIS GAS D'ALBINISME RENCONTRÉS EN SUISSE DANS LA RACE BRUNE DES ALPES
}

\author{
W. WEBER et J. J. LAUVERGNE \\ Institut für Tierzucht, Universität Bern; \\ Station centrale de Génétique animale, \\ Centre national de Recherches zootechniques, Jouy-en-Josas (Seine-et-Oise)
}

SOMMAIRE

Les trois cas d'albinisme observés en race Brune des Alpes de Suisse s'apparentent aux cas déjà décrits dans cette race. Il est fort probable qu'il s'agit de la réapparition sporadique de la même mutation; cette proposition reste toutefois à prouver.

\section{INTRODUCTION}

Chez les Mammifères, les mutations au locus albinos (C) sont très répandues. Leur principale caractéristique est de faire disparaître plus ou moins complètement, selon l'allèle, les pigments mélaniques des téguments, phanères et yeux.

Elles forment, en général, des séries alléliques où l'ordre de dominance est inverse de la capacité de réduction des pigments. Cette capacité de réduction s'exerce plus sur la phœomélanine jaune que sur l'eumélanine noire.

Dans beaucoup d'espèces la constitution et la vitalité des albinos sont normales ou sub-normales, même s'il s'agit de l'allèle provoquant la disparition totale du pigment.

On sait, par ailleurs, que le locus $\mathrm{C}$ contrôle l'activité d'une enzyme-oxydase, la tyrosinase.

Ce caractère biochimique n'est toutefois pas nécessaire pour identifier le locus C : l'absence d'anomalies concomitantes jointe au fait que les pigments oculaires sont 
réduits, que la phœomélanine disparaît d'abord et que la récessivité est de règle, suffit, en général, à le distinguer des éventuelles " mimiques " ("blancs dominants ", ou " pink eye ", par exemple).

Ces dernières années on a signalé, en race bovine Brune des Alpes, l'existence de veaux dépigmentés que nous avons classés comme albinos, en tenant compte des critères énumérés ci-avant.

Bien que nous n'ayons pas eu la possibilité d'en entreprendre l'étude poussée, nous pensons qu'il n'est pas inutile d'en signaler l'existence par une courte note.

\section{DESCRIPTION}

- Ier cas: en I958, à Andeer (Canton des Grisons), est né un veau de sexe mâle, totalement dépigmenté, à yeux et téguments roses.

Les parents, de race Brune des Alpes, étaient normalement colorés.

(Renseignements communiqués par le Dr Vét. SAURER.)

- $2^{\mathrm{e}}$ cas: en I959, à Malters (Canton de Lucerne), est né un veau de sexe femelle totalement dépigmenté, à yeux et téguments roses. L'animal a vécu 4 semaines manifestant une vigueur et une croissance normales; puis il a été vendu en boucherie.

Les parents, de race Brune des Alpes, étaient normalement colorés mais on ne possède aucun renseignement généalogique les concernant.

(Renseignements communiqués par le $D^{r}$ Vét. Nussbaumer.)

- $3^{\mathrm{e}}$ cas: en I960, à Kallnach (Canton de Berne), dans l'élevage Köhli-Köhli, est né un veau de sexe mâle que nous avons pu examiner alors qu'il était âgé de trois semaines. On a noté une absence totale de pigment dans la peau, les muqueuses, les poils et les onglons ; l'iris et le fond de l'œil paraissaient roses.

L'animal a vécu un an sans manifester de photophobie, aux dires de l'éleveur ; sa croissance était normale; il a été vendu en boucherie.

Les parents, de race Brune des Alpes, étaient normalement colorés sans qu'on possède de renseignements généalogiques les concernant.

Dans les trois cas évoqués, il s'agissait d'un albinisme total dont la nature héréditaire peut être présumée monofactorielle, autosomale et récessive.

\section{DISCUSSION}

L'albinisme a été décrit à plusieurs reprises dans l'espèce bovine. Les deux races oì il est le mieux connu sont la race Frisonne Pie Noire et la race Brune des Alpes.

- En Frisonne Pie Noire l'albinisme s'est manifesté aux U. S. A. (DETLEFSEN, I920; Colse, I929), aux Pays-Bas (Kroon et Plank, I932; Gotrnk et coll., I955), en Allemagne (Krailinger, I937) et au Japon (MAtsumoto et Tsutsumi, I954). 
Aux U. S. A. et au Japon, il s'agissait d'un albinisme incomplet : avec 1'âge se développait une coloration au niveau de l'iris qui devenait grisâtre et au niveau des taches normalement noires de la robe qui donnaient un dessin particulier appelé " ghost pattern " (patron spectre) ; le déterminisme apparaissait autosomal récessif et l'origine de la mutation semblait unique (Cole et coll., I934; Petersen et coll., 1944; Matsumoto et 'Tsutsumi, I954; Kumazaki et Mori, i962).

Dans les albinismes décrits par KROON et PLANK (I932) et KRALLINGER (I937), l'iris était pigmenté en bleu grisâtre mais on n'observait pas le " patron spectre "; la récessivité autosomale était prouvée mais sans qu'on puisse préciser une éventuelle origine commune. Les renseignements que donnent GoTnk et coll. (I955) sur des cas d'albinisme apparaissant sporadiquement aux Pays-Bas sont encore plus succincts.

- En race Brune des Alpes l'albinisme a été signalé 3 fois : par KELI.ER (I93I) en variété Montafon, par CARSTEns et coll. (I934) en variété Algäu chez des animaux inscrits au H. B. wurtembourgeois, enfin par ScHLEGER (I959) en variété Murbodner, dans un élevage de Basse-Autriche.

Les trois descriptions concordent : absence totale de pigment dans la peau, les muqueuses, le poil et les yeux. Les veaux albinos, bien que manifestant de la photophobie ont un développement normal (CARSTENS et coll., I934; SCHLEGER, I959); avec l'âge n'apparaît aucune pigmentation, au contraire de ce qui se passait chez les veaux frisons des U. S. A. et dı Japon. La monofactorialité autosomale récessive était démontrée par des expérience s de croisement dans le cas étudié par CaRSTENS et coll., I934 ; elle semblait également s'appliquer aux cas de KELLER (I93I) (les deux parents étaient normalement pigmentés) et de ScHLEGER (r959) (parents normaux et étroitement apparentés : croisement mère $\times$ fils).

Phénotypiquement, les cas que nous avons pu examiner en race Brune des Alpes s'apparentent aux cas déjà décrits dans cette race, bien qu'il faille faire une réserve puisqu'on n'a pu suivre le devenir de la couleur avec l'âge.

Génétiquement la récessivité autosomale que nous constatons correspond à celle de tous les albinos déjà étudiés.

L'appartenance de nos sujets à la iace Brune des Alpes où les liens de parenté de toutes les variétés avec des souches originaires de Suisse sont bien connus, laisse à penser que l'apparition de 6 mutations distinctes pour l'albinisme total au sein de cette race, est fort peu probable. Il reste à démontrer l'existence de liens entre tous ces cas, tâche ardue vu leur dispersion dans le temps et l'espace.

Rę̧u pour publication en février 1964 .

\section{SUMMARY}

THREE CASES OF AIJIINISM FOUND IN "SWISS RROWN" CATTLE

The three cases of albinism seen in Broten cattle from Switzerland are allied to cases already described in this breed. It is very probable that they are sporadic reappearances of the same mutation. This proposal still remains to be proved. 


\section{ZUSAMMENFASSUNG}

\section{DREI FÄLLE VON ALBINISMUS, BEIM SCHWEIZERISCHEN BRAUNVIEH}

Es werden drei Fälle von Albinismus bei der schweizerischen Braunviehrasse beschrieben. Es handelt sich wahrscheinlich um das sporadische Auftreten derselben Mutation, eine allerdings nicht bewiesene Annahme.

\section{RÉFÉRENCES BIBLIOGRAPHIQUES}

Carstens P., Mehner A., Prüfer J., i934. Untersuchungsergebnisse über das Auftreten und Verhalten von Albinos beim Braunvieh. Züchtungskunde, 9, 399-4I I.

Cole L. J., 1929. Albino cattle furnish interesting problem in inheritance. Wis. Agr. Exp. Sta. Bull., $n^{\circ} 405,65-66$.

Cole L. J., Van Lone E. E., Joltansson I., 1934. Albinotic dilution of color in cattle. J. Hered., 25. $145^{-1} 5^{6 .}$

DetlefseN J. A., I920. A herd of albino cattle. J. Hered., 11, 378-379.

Gotink W. M., De Groot Th., Stegenga Th., i955. Défauts héréditaires dans l'élevage bovin (en néerlandais). Landbouivk. Tiidschr., 's. Grav., 67, 629-672.

Keller K., I931. Die Bedeuntung der modernen Vererbungslehre für die praktische, namentlich die landwirtschaftliche Tierzucht. Zïchtungskunde, 6, 44-58.

Krallinger N. F., 1937. Über die Ausspaltung weisser Kälber in einer schlesischen Herde des Schwarzbunten Niederungsviehs. Züchtungskunde, 12, 273-276.

Kroon H. M., Plank G. M. v. d., 1932. Einige subletale Faktoren bei Haustieren in den Niederlanden. Biol. Gen., 8. 213-218.

KUMAZAKI K., MoRI J., I962. Albino cattle of the Holstein-Friesian breed appearing in the western region of Japan (en japonais avec résumé anglais). Jap. J. Zootech. Sci., 32, 362-368.

Matsumoto K., Tsutsumi Y., I954. Albino calves found in the Holstein-liriesian breed in Japan, with notes on the hair structure (en japonais avec résumé anglais). Jap. J. Zootech. Sci., 25, I51-154.

Petersen W. E., Gilmore L. O., Fitch J. B., Winters L. M., 1944. Albinism in cattle. J. Hered., 35, I35-I 44 .

SChleger W., 1959. Auftreten eines Albinokalbes bei der Murbodnerrasse. Wien. Tieräztl. Mschr., 46, 196-199. 